\title{
Membrane flow patterns in multicomponent giant vesicles induced by alternating electric fields $\uparrow$
}

\author{
Margarita Staykova, Reinhard Lipowsky and Rumiana Dimova* \\ Received 11th July 2008, Accepted 27th August 2008 \\ First published as an Advance Article on the web 10th September 2008 \\ DOI: $10.1039 / b 811876 k$
}

\begin{abstract}
Alternating electric (AC) fields induce circular patterns of lipid transport in membranes of giant vesicles. The flow is visualized by fluorescently labelled lipid domains.
\end{abstract}

Electric fields induce various electrokinetic phenomena, which have found wide application in micro- and nano-technologies in the past decade. Electro-neutral particles (droplets, bubbles, lipid vesicles, solid beads) suspended in a medium of different polarizability acquire charge at their surfaces when exposed to electric fields. The interaction of the fields with the surface charges may result in particle kinetics, electro-osmotic fluid flow or a combination of both. ${ }^{1}$

Particle kinetics in electric fields arise from the interaction of the field with the field-induced dipole moment. Depending on the field geometry, freely suspended particles may translate, orient, rotate, deform, etc. These kinetic phenomena are widely used in biology and biotechnology as methods for cell or membrane characterization ${ }^{2}$ and micro-manipulation. ${ }^{3}$

Electro-osmotic flows in the vicinity of polarizable objects are driven by the displacement of the free induced surface charges by the lateral component of the electric field. This phenomenon has been termed "induced-charge electro-osmosis" (ICEO) ${ }^{1}$ and has drawn significant attention because of its vast application potential in microfluidics. ICEO has been reported and theoretically interpreted for the first time by Taylor in relation to the deformation of a droplet in a homogeneous steady electric field. ${ }^{4}$ The flow outside the drop is symmetric on both sides facing the electrodes and is directed from the poles (the points nearest to the electrodes) to the equator. A similar flow pattern has been theoretically predicted for solid polarizable particles in AC fields. ${ }^{5}$ More recently, external fluid flows have been studied for various geometries and conditions ${ }^{6}$ but, to our knowledge, no experimental or theoretical studies on ICEO effects on lipid membranes have been pursued. Here, we report electro-osmotic lipid flow patterns induced by AC fields. We used giant vesicles, tens of micrometres in size, which are very suitable for direct microscopy observation of the influence of electric fields on membranes. ${ }^{2 c}$

Lipid vesicles provide biomimetic models for cell membranes and have many biotechnological applications, e.g. as drug delivery systems, micro-reactors, etc. Since many techniques for cell manipulation, gene therapies and cancer treatment use electric fields as an external perturbation, it is important to understand the nature of

Max Planck Institute of Colloids and Interfaces, Science Park Golm, 14424 Potsdam, Germany. E-mail: Rumiana.Dimova@mpikg.mpg.de; Fax: +49 331567 9615; Tel: +493315679612

$\dagger$ Electronic supplementary information (ESI) available: Vesicle preparation procedure, numerical calculations and confocal microscopy movies of domain motion. See DOI: 10.1039/b811876k their effect on the membrane at the molecular level. Here, we implement fluorescent markers to visualize the flow patterns of the lipid movement within the membrane of a vesicle subjected to an AC field.

The behaviour of lipid vesicles in AC fields is determined to a large extent by the specific electric and mechanical properties of their membranes. The lipid membrane can be considered as a thin fluid layer hydrophobically confined at the surface of the vesicle. At low field frequencies the membrane behaves as an electric insulator between the inner and the outer electrolyte solutions. Charges accumulate on both membrane interfaces and the membrane is capacitively charged. At higher frequencies (e.g. above $1 \mathrm{kHz}$ ), the membrane capacitor becomes short-circuited and the induced surface charge density depends solely on the conductivities of the inner and outer vesicle media. Hydrodynamically, the membrane behaves as a two-dimensional incompressible fluid. To keep the surface area constant the lipid bilayer develops tension under forcing. Under homogeneous electric fields, an axially symmetric gradient in the membrane tension builds up, which effectively counterbalances the lateral component of the electric stress; see the ESI. $\dagger$ As a result, an electro-osmotic membrane flow in lipid vesicles is not expected. ${ }^{7}$ However, in most chambers and conditions used for electric manipulation, vesicles, cells or other particles are located close to the chamber bottom between the electrodes, or among other cells or vesicles, or in the immediate vicinity of an electrode, ${ }^{8}$ thus experiencing an inhomogeneous electric field. This inhomogeneity is even more pronounced in chambers for micro-manipulation where the electrodes are imprinted on a solid substrate..$^{3 b}$ In this study, we show for the first time that even weakly inhomogeneous AC fields may induce a pronounced membrane flow in giant lipid vesicles.

We prepared giant lipid vesicles from dioleoylphosphatidylcholine (DOPC), dipalmitoylphosphatidylcholine (DPPC) and cholesterol (Chol) in molar ratios of $5.33: 2.66: 2,2.66: 5.33: 2$ or $4.8: 3.2: 2$ DOPC : DPPC : Chol, using the electroformation method; ${ }^{9}$ see the ESI. $\dagger$ A small fraction of $N$-(lyssamine rhodamine B sulfonyl)dipalmitoylphosphatidylethanolamine (Rh-DPPE) (0.1 molar $\%)$ was added to the lipids for fluorescence microscopy observation. The vesicles were prepared in 0.01 to $0.1 \mathrm{M}$ sucrose and subsequently diluted in isotonic glucose solution to prevent osmotic stress and to make them sediment to the bottom of the observation chamber. Up to about $3 \mathrm{mM} \mathrm{NaCl}$ was added to the glucose solution to adjust the vesicles' external conductivity. The internal conductivity was always in the range $2-5 \mu \mathrm{S} \mathrm{cm}$, whereas the external one was varied between 2-5 and $300 \mu \mathrm{S} \mathrm{cm}^{-1}$. At room temperature, the ternary lipid mixture (with the above-mentioned composition) spontaneously separates in liquid-ordered $\left(l_{o}\right)$ and liquid-disordered $\left(l_{d}\right)$ phases, leading to the formation of $1_{o}$ and $1_{d}$ domains on the vesicles. ${ }^{10}$ 
Rh-DPPE preferentially partitions in the $1_{d}$ domains. The ternary lipid composition and the temperature conditions determine the size of the domains. An optimal condition for our observation was provided by many small $l_{\mathrm{o}}$ domains, appearing as dark circular patches of 5-10 $\mu \mathrm{m}$ diameter on a red background. The lipid domains turned out to be very suitable markers for studying membrane fluid dynamics induced by different stresses such as electric, hydrodynamic (e.g. shear flow), mechanic, etc.

The vesicles were exposed to AC fields of moderate field strengths $\left(100-500 \mathrm{~V} \mathrm{~cm}^{-1}\right)$ and intermediate frequencies of $10 \mathrm{kHz}$ to $20 \mathrm{MHz}$. The working chamber consists of two parallel cylindrical electrodes, $200 \mu \mathrm{m}$ in diameter, at a distance of $500 \mu \mathrm{m}$. The distance of the electrodes from the bottom of the chamber, a glass surface, is $c a$. 2-5 $\mu \mathrm{m}$. Because of sedimentation, the vesicles are exposed to the field close to the bottom of the chamber. The observations were done with a confocal microscope at various focal planes across the vesicles, whereby the pinhole was widely open to allow for a large scan depth, see the ESI. $\dagger$ Appropriate for observation are single spherical vesicles located in the middle between the electrodes, with diameters between 70 and $150 \mu \mathrm{m}$.

When the vesicles were exposed to AC fields, we observed some familiar electrokinetic phenomena. Under the conditions of our experiments, the vesicles experience negative dielectrophoresis, manifested as repulsion from the high field intensity regions, i.e. from the electrodes and the solid glass substrate. At the same time they deform, assuming an oblate shape as previously reported. ${ }^{2 c, 11}$ In addition, an interesting new phenomenon is observed, namely circular lipid flow patterns within the vesicle membrane.
The membrane flow is studied by following the motion of the lipid domains on the vesicle. Fig. 1 provides snapshots of this motion on the top and bottom vesicle parts, as well as schematic illustrations of the flow lines; movies showing the domain displacement are available in the ESI. $\uparrow$ The flow velocity reaches about $30 \mu \mathrm{m} \mathrm{s}^{-1}$ corresponding to laminar flows. It depends on the field conditions and on the location within the vesicle surface. In all experiments, the lipid transport is faster at the bottom part of the vesicle (closer to the glass).

The lipid dynamics on the vesicle surface is organized in four symmetric quadrants, each extending from the lower to the top part of the vesicle as illustrated in Fig. 1f. The motion follows concentric closed trajectories with the highest velocity at the periphery of each quadrant and at the vesicle bottom. Towards the quadrant centres, the velocity decreases creating stagnation points, where the motion stops. Note that these points appear slightly below the middle plane of the vesicle. For a vesicle with a radius of $75 \mu \mathrm{m}$, the time period, during which a domain completes a full cycle of its trajectory, varies between about $10 \mathrm{~s}$ when located close to a quadrant centre, and $90 \mathrm{~s}$ when in the quadrant periphery. Additionally, there are two other stagnation points at the top and the bottom of the vesicle, where membrane motion is slowed down by the balance of oppositely directed forces. For smaller vesicles with diameters less than $60 \mu \mathrm{m}$, the lipid flow does not fully extend to the upper hemisphere and the stagnation points in the quadrants are located even closer to the vesicle bottom.

We have further attempted to characterize the dependence of the lipid transport on the field parameters and the media properties. At higher field strength the motion is faster. At the same time however,

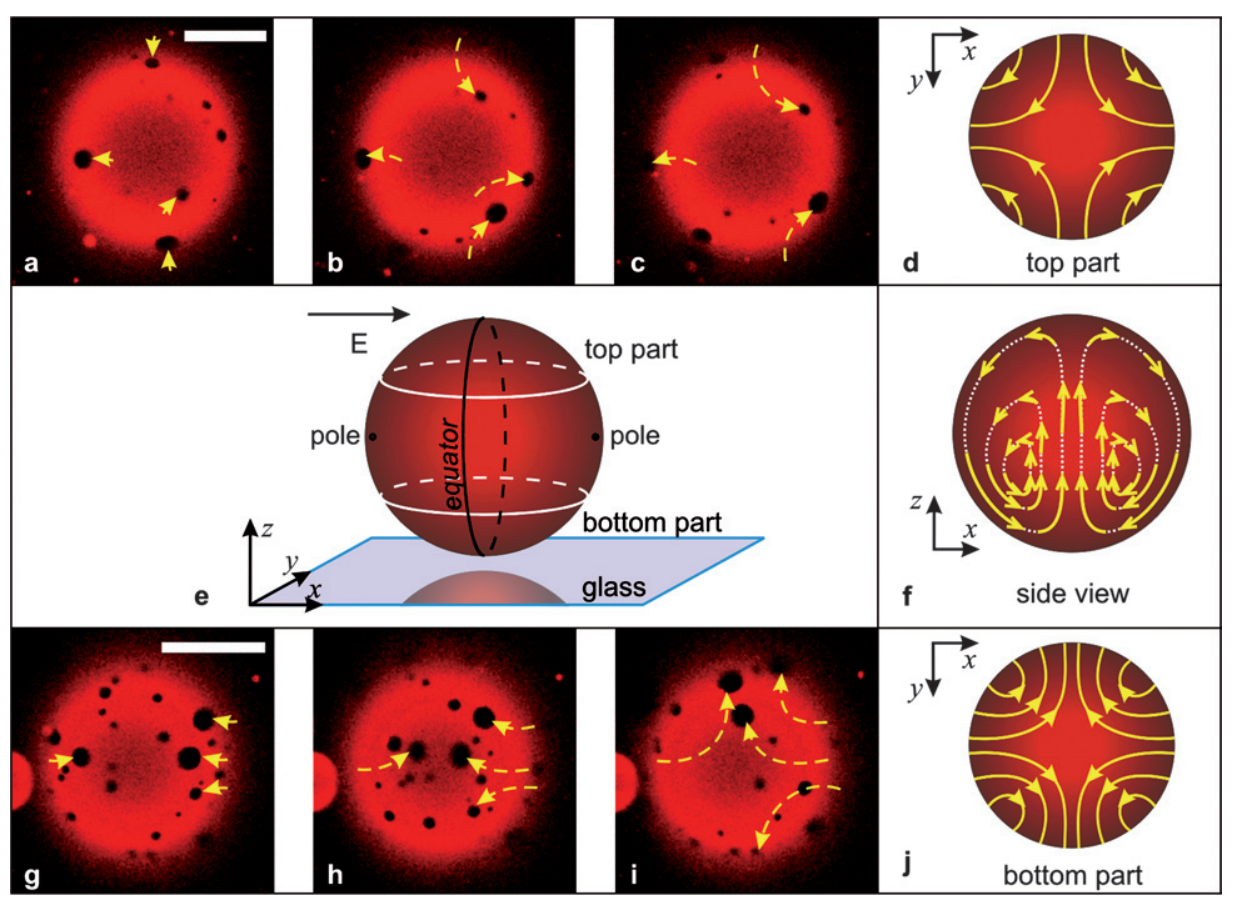

Fig. 1 Confocal micrographs illustrating the membrane flow on the top (a-c) and bottom ( $\mathrm{g}-\mathrm{i}$ ) parts of a giant vesicle $(\sim 150 \mu \mathrm{m}$ in diameter) induced by an AC field ( $\left.360 \mathrm{~V} \mathrm{~cm}^{-1}, 80 \mathrm{KHz}\right)$, at external conductivity of $250 \mu \mathrm{S} \mathrm{cm}^{-1}$. The vesicle was prepared from a mixture of $4.8: 3.2: 2 \mathrm{DOPC}:$ DPPC : Chol. The time between the consecutive snapshots is approximately $1.3 \mathrm{~s}$ for a-c and $6.5 \mathrm{~s}$ for $\mathrm{g}-\mathrm{i}$. The yellow dashed arrows indicate the trajectories of selected domains. The scale bars correspond to $50 \mu \mathrm{m}$. The vesicle is located close to the bottom of the observation chamber as illustrated in (e), where the vesicle top and bottom parts, the poles and the equator as well as the field direction are indicated. The top, side and bottom view of the flow lines are sketched in (d), (f) and (j), respectively. The length of the arrows in (f) roughly corresponds to the flow velocity. 
the negative dielectrophoretic force lifting the vesicles away from the glass substrate increases. The latter effect depends on the vesicle weight. For lighter vesicles, containing $0.01 \mathrm{M}$ sucrose solution, the lipid motion in the membrane at first increases with increasing field strength and afterwards gradually decreases as they drift away from the bottom of the chamber. When the vesicles are far away from the glass substrate, the field is more homogeneous and the membrane motion may terminate completely even though the vesicle deformation is preserved. Vesicles with diameters between 70 and $150 \mu \mathrm{m}$, containing $0.1 \mathrm{M}$ sucrose, are heavy and are not lifted significantly for fields up to $400 \mathrm{~V} \mathrm{~cm}^{-1}$. The lipid flow is also enhanced by the external conductivity whereas it is very slow in salt-free external media.

Interesting behaviour was observed when the field frequency was varied; see the ESI. $\dagger$ At frequencies lower than about $1 \mathrm{MHz}$, the motion in the circular trajectories is directed downwards past the poles and upwards along the equator as sketched on Fig. 1f. With increasing frequencies the velocity of the motion gradually decreases and reverses its direction at around 3-5 $\mathrm{MHz}$.

In order to get some insight into the onset of the membrane flow, we calculate the tangential force arising from the inhomogeneous electric field. For this purpose, we use a commercially available modelling package COMSOL (Multiphysics, Germany). Since the frequencies used in the experiments were well above $1 \mathrm{kHz}$ and the internal solution has a very low conductivity, the induced charges arise from the external solution. Thus, we can use with good approximation the equivalent body model; for details see ESI. $\uparrow$ According to it, the vesicle can be represented as a homogeneous spherical object with an effective permittivity and conductivity. ${ }^{12}$ Fig. 2 presents the calculated distribution of the electric field around a vesicle at a frequency of $100 \mathrm{kHz}$; see the ESI $\dagger$ for details on the model parameters. The field strength exhibits minima at the vesicle poles and higher values at the equator. In addition, the vicinity of the glass causes asymmetric field distribution on the surface of the vesicle. The field is much higher at the lower region facing the glass than at the vesicle top. Such field distribution agrees well with our experimental findings for oblate deformation (enhanced electric pressure at the poles) and negative dielectrophoresis (high field strength at the bottom part pushes the vesicle upwards). Note that the out-of-sphere deformation of the vesicle is not taken into account in the calculations
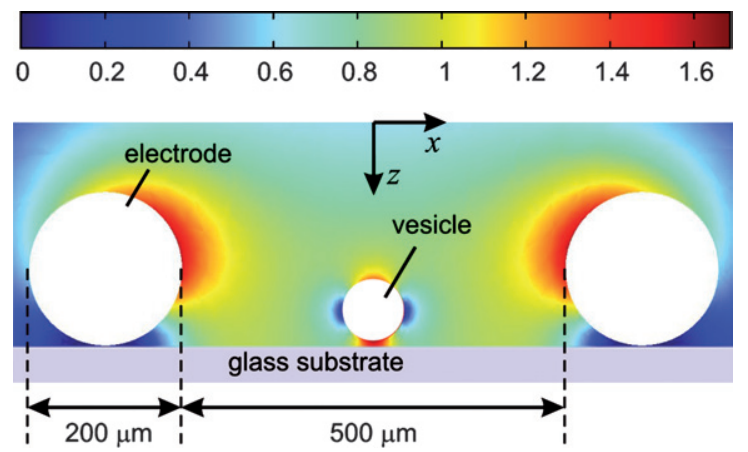

Fig. 2 Electric field distribution at $100 \mathrm{kHz}$ in a cross section of the chamber, passing through the centre of the vesicle. The vesicle is $40 \mu \mathrm{m}$ in radius and is located at $8 \mu \mathrm{m}$ above the glass. The media conductivity is $300 \mu \mathrm{S} \mathrm{cm}^{-1}$. The field inside the vesicle is not calculated. The data are rescaled with the strength of a field, which would be induced between two parallel planar electrodes at a distance of $500 \mu \mathrm{m}$. since it is relatively small and does not influence the results qualitatively.

Given the field distribution, we calculate the lateral electric stress (surface force density) on the membrane as the product of the fieldinduced free charge density at the vesicle surface and the tangential component of the electric field. ${ }^{4}$ In homogeneous fields (electrodes and vesicle located high above the chamber bottom), the lateral stress distribution is axially symmetric; see the ESI. $\dagger$ It exhibits minima at the poles (zero tangential electric field) and at the equator (minimum induced free charge). The maxima are located at $\theta= \pm \pi / 4$, where $\theta$ is the angle between the radial vector of the vesicle and the field vector. The membrane tension adapts to the symmetric lateral stress. As a result no lipid flow is expected as observed for vesicles far away from the solid substrate.

The inhomogeneous electric field distribution for vesicles close to the glass (and generally in chambers for micro-manipulation) breaks the symmetry in the electric stress distribution between the lower and the upper hemispheres of the vesicle; see Fig. 3. Overall, the vesicle experiences significant stress at $\theta= \pm \pi / 4$, as in the homogeneous case, but it is additionally enhanced in the vicinity of the solid substrate. As a result, a non-uniform and non-symmetric membrane tension builds up. It triggers lipid flow towards the regions of highest tension, in analogy to Marangoni flow. The gradients in the tension are strongest in the regions facing the electrodes. There the membrane starts flowing downwards. At the bottom of the vesicle, where two oppositely directed flows meet, the membrane material is pushed upward along the equator for reasons of mass conservation. At the top, the flow splits in two directions downwards to the two poles due to gravity. These are the principal flow lines as sketched in Fig. If for a field frequency up to $1 \mathrm{MHz}$. Interestingly, the frequency at which we observe reversal of the flow direction, i.e. around $3 \mathrm{MHz}$, coincides with the Maxwell-Wagner frequency, above which the polarization of the vesicle is determined by the media permittivities. Thus, the reversal of the flow direction may be due to the difference between the permittivities of the glucose and the sucrose solutions. The flow in the membrane is expected to induce fluid motion in the inner and

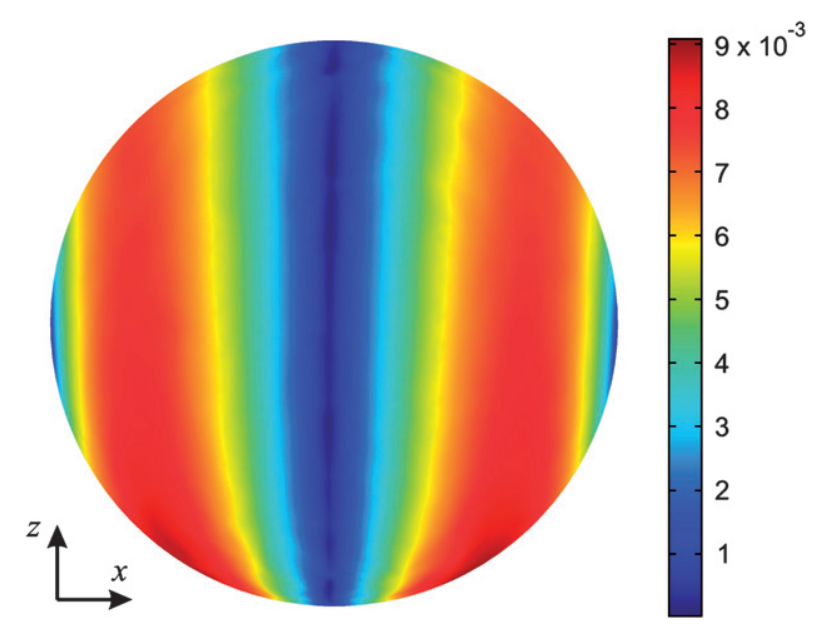

Fig. 3 Lateral electric stress magnitude on the surface of a vesicle as defined in the text (for parameters see Fig. 2). The applied electric field is directed along the $x$ axis. The calculated values of the stress have been divided by $\varepsilon_{\mathrm{ex}} E_{0}^{2}$ corresponding to a homogeneous electric field $E_{0}$ acting on a homogeneous external medium with dielectric constant $\varepsilon_{\mathrm{ex}}$. 

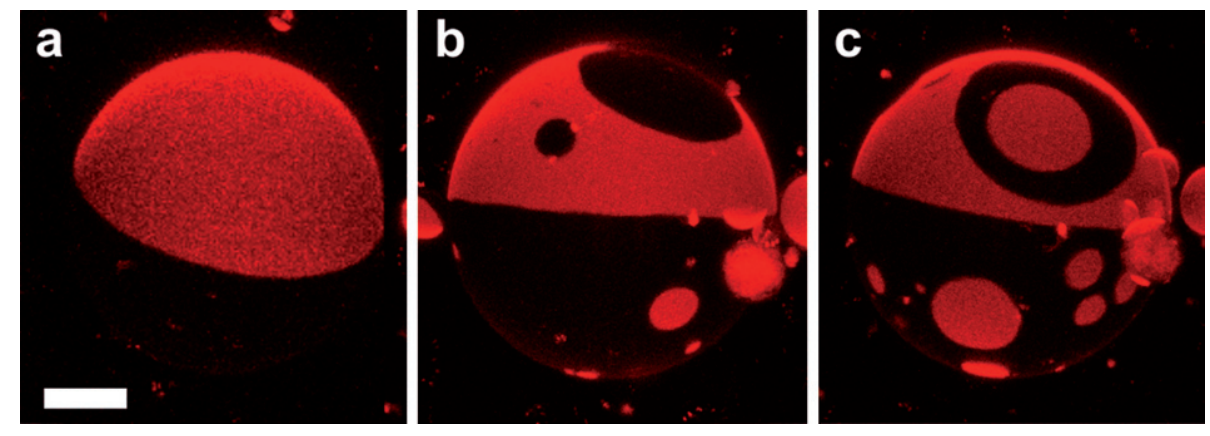

Fig. 4 3D confocal scans of the lower vesicle hemisphere illustrating lipid mixing induced by AC field $\left(80 \mathrm{kHz}, 500 \mathrm{~V} \mathrm{~cm}^{-1}\right)$, at an external conductivity of $250 \mu \mathrm{S} \mathrm{cm}^{-1}$. The vesicle ( $\sim 95 \mu \mathrm{m}$ diameter) was prepared from a mixture of $2.66: 5.33: 2$ DOPC : DPPC : Chol. Before applying the field (a), the vesicle has only two domains, which break apart after continuous field exposure of $2 \mathrm{~min}$ (b) and $3 \mathrm{~min}$ (c). The scale bar corresponds to $25 \mu \mathrm{m}$.

outer media, via viscous coupling, but we did not attempt to visualize it.

The above mechanism is only an initial conjecture explaining the observed lipid flow lines based on the numerical calculations of the electric field and lateral stress. It does not account for possible hydrodynamic effects and shielding from neighbour vesicles.

The AC field-induced membrane flow may possibly find significant application in microfluidic technologies. Giant vesicles in inhomogeneous $\mathrm{AC}$ fields or in hydrodynamic flows mimicking, e.g., the situation of red blood cells in capillaries may be used as nano-reactors for fluid manipulation, i.e. displacing, mixing, trapping, etc. To demonstrate lipid mixing, we performed experiments where lipid vesicles composed of only one $l_{\mathrm{o}}$ and one $1_{\mathrm{d}}$ domain, are exposed to an AC field for a certain period of time. One example is shown in Fig. 4. The field-induced membrane flow causes domain fission leading to the appearance of a large number of smaller domains; see also the movies in the ESI. $\dagger$ In a fission process, there is a competition between the domain line tension and the local lateral force on the membrane. Therefore, for low field strengths or low external salt concentrations, the lateral stress is insufficient to overcome the line tension, and the domains only deform. For sufficiently strong membrane flows, the number of domains grows with the time of exposure. This, on the other hand, increases the probability of domain encounter and fusion. Domain fusion counterbalances the fission and therefore, after a certain time, the domains will reach a stationary state characterized by a certain size distribution. Since the membrane flow follows a certain pattern, the lipid mixing is not random. Domain fusion and fission are observed only at the bottom part of the vesicle, where oppositely directed flows meet and are deflected. At the stagnation points in the centres of the circular trajectories (see Fig. 1f) the membrane material may be trapped and a certain quasi-stable domain configuration surrounded by fast flowing domains may be observed (Fig. 4c). After switching the field off, the domains are not in equilibrium and slowly fuse back into two big domains.

The AC field-induced lipid flow as demonstrated here for giant vesicles with intramembrane domains opens a new direction of research. Such membrane labelling allows visualization of lipid displacement and we believe that this approach will be helpful for studies on membrane behaviour in vesicles subjected to shear flows ${ }^{13}$ or mechanical stresses. Investigating electric field-induced membrane flows may also lead to a deeper understanding of a widely used but poorly understood technique for giant vesicle preparation, namely the electroformation method. ${ }^{9}$ Finally, we expect to observe other interesting electro-osmotic phenomena by studying the influence of the electric field geometry and the shape of the vesicle on the flow pattern.

\section{Acknowledgements}

MS thanks N. Bezlyepkina, R. Knorr, S. Aranda and Y. Li for experimental support. We acknowledge P. Vlahovska for fruitful discussions and critical reading of the text.

\section{Notes and references}

1 T. M. Squires and M. Z. Bazant, J. Fluid. Mech., 2004, 509, 217-252.

2 (a) H. Schwan, Blut, 1983, 46, 185-197; (b) J. Yang, Y. Huang, X. Wang, X-B. Wang, F. Becker and P. Gascoyne, Biophys. J., 1999, 76, 3307-3314; (c) R. Dimova, K. Riske, S. Aranda, N. Bezlyepkina, R. Knorr and R. Lipowsky, Soft Matter, 2007, 3, 817-927.

3 (a) U. Zimmermann, Electromanipulation of Cells, CRC Press, Boca Raton, FL, 1996; (b) J. Voldman, Annu. Rev. Biomed. Eng., 2006, 8, 425-454.

4 G. I. Taylor, Proc. R. Soc. London, Ser. A, 1966, 291, 159-166.

5 (a) V. A. Murtsovkin, Colloid J. (Transl. of Kolloidn. Zh.), 1996, 58, 341-349; (b) G. Yossifon, I. Frankel and T. Miloh, Phys. Fluids, 2007, 19, 068105 .

6 (a) A. Ramos, H. Morgan, N. G. Green and A. Castellanos, J. Colloid Interface Sci., 1999, 217, 420-422; (b) M. Z. Bazant and T. M. Squires, Phys. Rev. Lett., 2004, 92, 066101.

7 (a) W. Helfrich, Z. Naturforsch., 1973, 29c, 693-703; (b) H. Hyuga, K. Kinosita and N. Wakabayashi, Jpn. J. Appl. Phys., 1991, 30, 2649-2656.

8 S. Lecuyer, W. Ristenpart, O. Vincent and H. Stone, Appl. Phys. Lett., 2008, 92, 104105 .

9 M. Angelova and D. Dimitrov, Faraday Discuss. Chem. Soc., 1986, 81, 303-311.

10 S. Veatch and S. Keller, Phys. Rev. Lett., 2002, 89, 268101.

11 S. Aranda, K. Riske, R. Lipowsky and R. Dimova, Biophys. J., 2008, 95, L19-L21.

12 H. Pauly and H. Schwan, Z. Naturforsch., 1959, 14b, 125-131.

13 (a) C. Vézy, G. Massiera and A. Viallat, Soft Matter, 2007, 3, 844 851; (b) M. Abkarian and A. Viallat, Soft Matter, 2008, 4, 653-657. 